\title{
Heavy flavour Production in ATLAS
}

\author{
Andreas SALZBURGER ${ }^{* \dagger}$ \\ CERN \\ E-mail: Andreas.Salzburger@cern.ch
}

We present a measurement of the inclusive and dijet differential cross sections of jets containing a $b$-hadron in proton-proton collisions at $\sqrt{s}=7 \mathrm{TeV}$ using data collected with the ATLAS detector. Jets are reconstructed using the anti- $k_{t}$ algorithm with jet radius parameter $R=0.4$. The presence of a displaced vertex from the decay of long-lived hadrons, or the presence of a muon with significant transverse momentum relative to the jet axis, is used to select a jet sample enriched in $b$-jets and the invariant mass of the charged particle tracks forming the vertex is fitted to extract the fraction of jets from $b$-quark production. The inclusive cross section is measured as a function of the jet transverse momentum, in the range $20<p_{\mathrm{T}}<260 \mathrm{GeV}$, and of rapidity, in the range $0<|y|<2.1$, such that jets are fully contained in the tracking detectors of ATLAS. The dijet cross section is measured in the same rapidity range as a function of the dijet invariant mass, extending up to $670 \mathrm{GeV}$. The resulting cross sections are compared with next-to-leading-order QCD predictions.

The 2011 Europhysics Conference on High Energy Physics-HEP 2011,

July 21-27, 2011

Grenoble, Rhône-Alpes France

\footnotetext{
* Speaker.

† On behalf of the ATLAS Collaboration.
} 


\section{Introduction}

The measurement of $b$-quark production cross sections at the LHC provides an essential test of perturbative QCD predictions at the energy frontier. Calculations of the $b$-quark production cross section have been performed at next-to-leading order (NLO). Matching these calculations to parton shower models produces final states that can be compared to experimental data. Experimentally, this can be assessed by measuring jets that contain a $b$-hadron, in the following referred to as $b$-jets. This note discusses the measurement of the inclusive $b$-jet cross section in the range $20<p_{\mathrm{T}}<260$ $\mathrm{GeV}$ and the $b \bar{b}$ dijet cross section extending up to a dijet invariant mass of $670 \mathrm{GeV}$ measured with the ATLAS detector [1] in proton-proton collisions at $\sqrt{s}=7 \mathrm{TeV}$. The measurements are compared to predictions using Pythia [2] using the ATLAS MC09 tune [3] and NLO predictions using POWHEG [4]. Further details of the analysis can be found in [5]. Results presented in this document are based on data collected between March and August 2010 at the ATLAS detector and correspond to an integrated luminosity of $3.0 \mathrm{pb}^{-1}$. The uncertainty of the integrated luminosity is $3 \%[6]$.

\section{Event selection and reconstruction}

Events were triggered using the Level-1 jet trigger, with the exception of the jets in the inclusive cross section analysis with a jet- $p_{\mathrm{T}}<40 \mathrm{GeV}$ for which the minimum bias trigger scintillators (MBTS) were used. The trigger efficiency was estimated to be above $98 \%$ in all $p_{\mathrm{T}}$ bins with negligible uncertainties. Triggered events were required to have a well reconstructed primary vertex with at least ten contributing tracks. This is essential for the $b$-jet identification which relies on primary and secondary vertex reconstruction. The jet energy has been calibrated to the standard ATLAS jet energy scale [7] with a systematic uncertainty between $5 \%$ and $9 \%$, depending on the transverse momentum of the jet. In both, data and simulation the $p_{\mathrm{T}}$-balance method [7] was applied to test the agreement with respect to each other. An additional systematic uncertainty of $2.5 \%$ to account for differences in the jet energy scale between $b$-jets and light jets was found and added in quadrature to the jet energy scale uncertainty. Due to the long lifetime of the $b$-quark, tracks in jets originating from $b$-quarks generally form a secondary vertex that is spatially separated by a few millimeters from the proton-proton collision vertex. The SV0 [8] $b$-tagging algorithm used in this analysis aims to reconstruct the displaced vertex from the charged decay products of the $b$-hadron. The discrimination between $b$-jets and light jets is done using the signed decay length significance $S=L / \sigma_{L}$, where $L$ defines the 3-dimensional distance between the reconstructed primary and sencondary vertex. In this analysis, a jet is considered $b$-tagged if it contains a secondary vertex reconstructed with the SV0 tagging algorithm with $S>5.72$, which gives an efficiency of $50 \%$ in simulated $t \bar{t}$ events. The efficiency of the $b$-tagging algorithm was measured from data with a sample of jets containing a muon, using the $p_{\mathrm{T}}^{r e l}$ method [8]. This method makes use of the distinctive differences in the relative transverse muon $p_{\mathrm{T}}$ distribution to the reconstructed jet axis for $b$ and light jets in the leptonic decay. The $p_{\mathrm{T}}^{r e l}$ method, however, is limited to a momentum range before the jets become strongly collimated. This is why for higher $p_{\mathrm{T}}$ jets the $b$-tagging correction factors between data and $\mathrm{MC}$ of the last accessible jet $p_{\mathrm{T}}$ bin is taken. The systematic uncertainties for this method are dominated by the template shapes and range between 10 to $15 \%$; they were 
doubled for the $p_{\mathrm{T}}$ bins that are not accessible due to jet collimation. The mistag rate of the SV0 algorithm was found by template fits to the secondary vertex mass and range between 0.2 and $1 \%$.

\section{Measurement of the inclusive $b$-jet and $b \bar{b}$ dijet cross section}

The definition of a $b$-jet production cross section requires a definition of a truth-level $b$-jet in Monte Carlo (MC). These are formed by clustering stable particles using the anti- $k_{t}$ [9] cluster algorithm with a radius parameter of $\mathrm{R}=0.4$. Truth-level $b$-jets are then defined by spatially matching the jet with generator-level hadrons: a $b$-jet is labelled as such if at least one $b$-hadron is found within a cone of $\Delta R=\sqrt{\Delta \eta^{2}+\Delta \phi^{2}}<0.3$ around the reconstructed jet direction.

In the inclusive cross section analysis, the number of $b$-jets in the final sample was determined by a fit to the secondary vertex mass, using MC templates for the $b, c$ and light quark contributions, respectively. Figure 1 shows the SV0 mass distributions and the different fitted MC templates before and after applying the $b$-tagging cut.
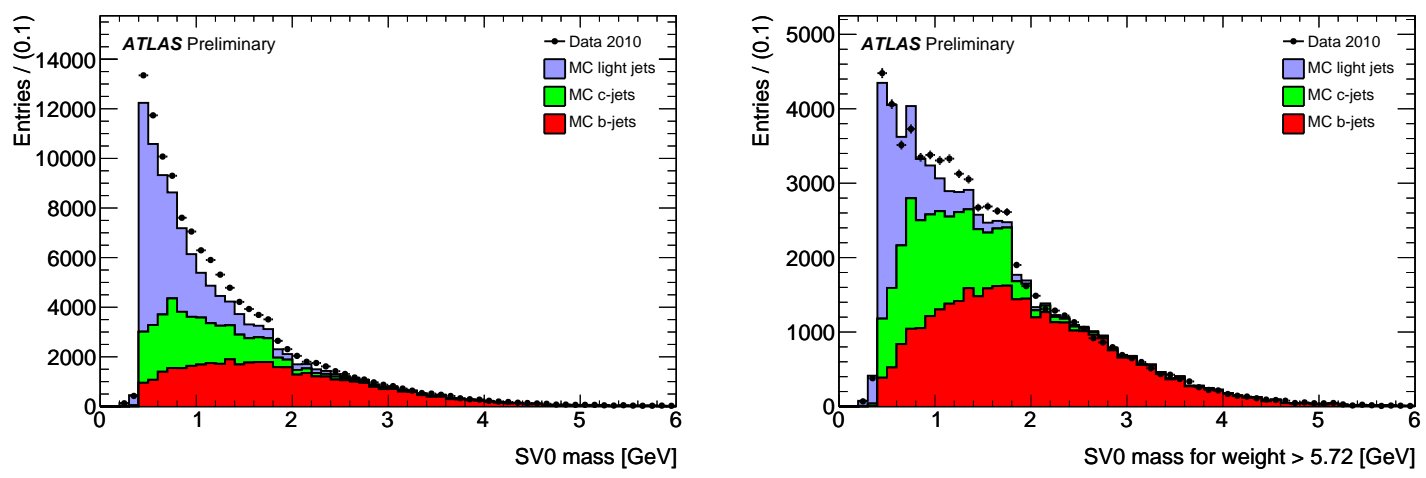

Figure 1: SV0 mass distribution in data and simulation (Pythia MC09) without a cut on the SV0 weight (left) and for jets with SV0 weight $S>5.72$ (right). In both plots, the MC is normalized to the number of jets in the data with at least one track passing the SVO

The double differential inclusive $b$-jet cross section is shown in Fig. 2 (left), where it is compared to results obtained with Pythia MC09 and POWHEG. Pythia MC09 is a leading-logarithmic parton shower generator, thus it is not expected to accurately predict the normalization. However, to compare the shape dependency, an adhoc scale factor of 0.52 has been used. This factor was found by normalizing the Pythia MC09 prediction to the same integrated cross section as the measurement. Both Pythia MC09 and POWHEG show a reasonable good agreement with the measured cross section, although both tend to fall more quickly with increasing $p_{\mathrm{T}}$. Using the inclusive jet cross section measured by ATLAS [10], a ratio of the $b$-jet to inclusive cross section can be built, which significantly decreases the systematic uncertainties: the uncertainty on the jet energy scale is almost canceled (except for the additional assigned component for $b$-jets). The measurement is then dominated by the uncertainties associated to the $b$-tagging efficiency and purity. The results can bee seen in Fig. 2 (right) for different rapidity bins. Again, the comparison to Pythia MC09 and POWHEG are added, where POWHEG shows an underestimation of the $b$-jet cross section by approximately $30 \%$ which corresponds to a $1 \sigma$ systematic effect. 

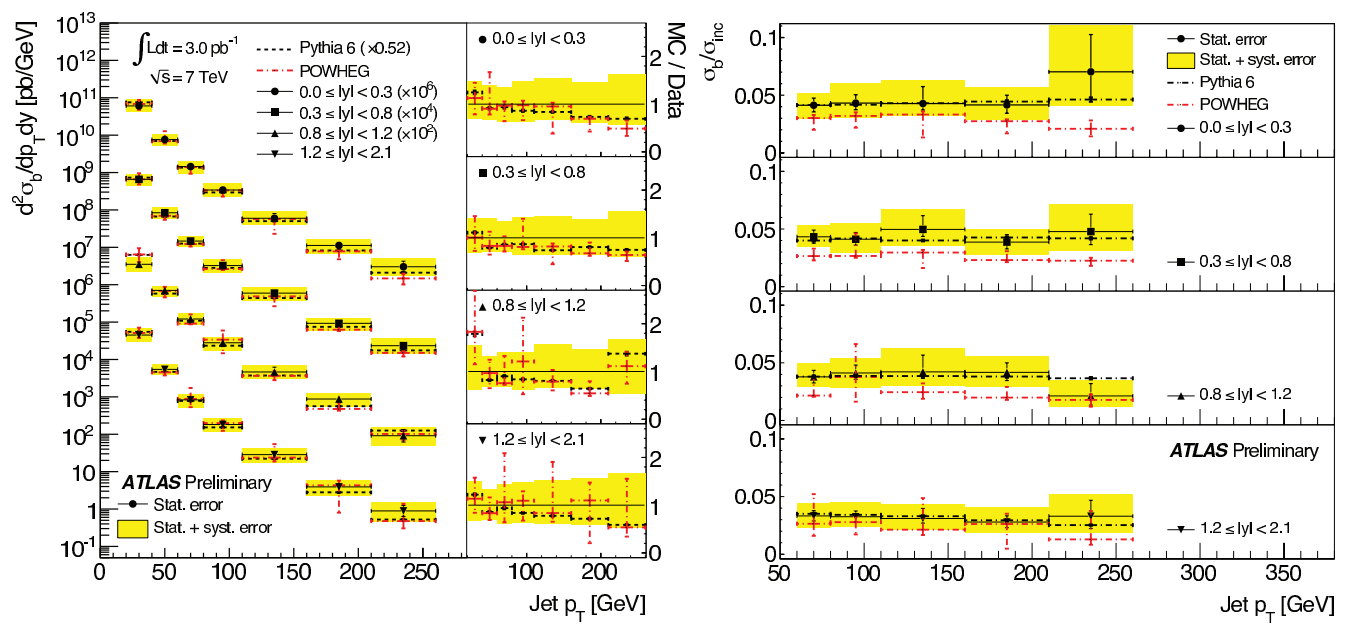

Figure 2: Double differential inclusive $b$-jet cross section (left). Ratio of inclusive $b$-jet cross section to the inclusive jet cross section (right). The data are compared to MC prediction of Pythia MC09 and POWHEG. The Pythia MC09 results are scaled by a factor of 0.52 .

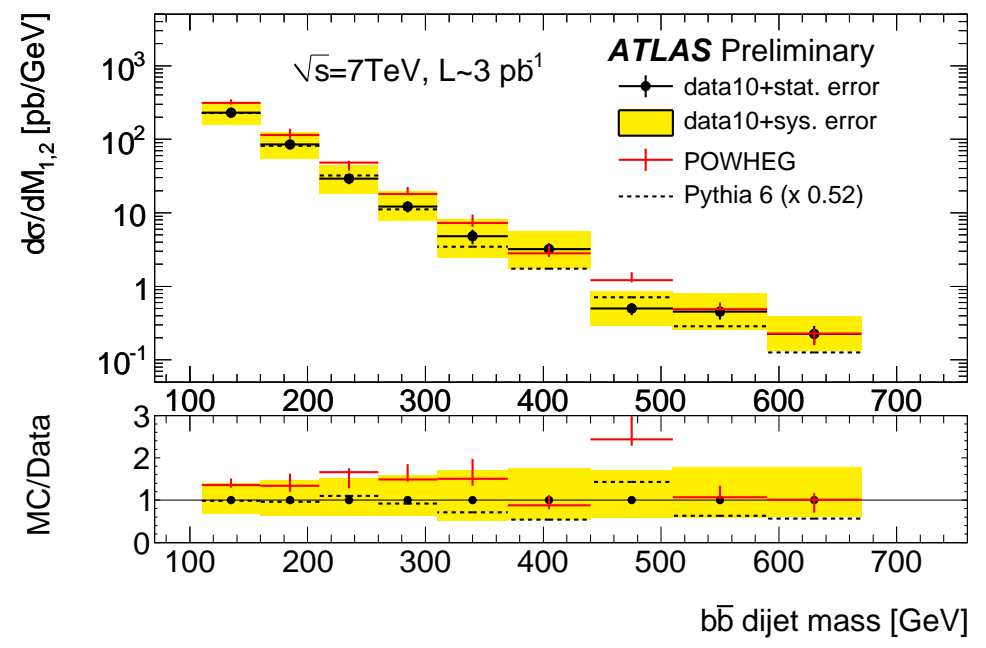

Figure 3: The $b \bar{b}$ dijet cross section as a function of dijet invariant mass for $b$-jets with $p_{\mathrm{T}}>40 \mathrm{GeV}$ and $|y|<2$.1. The data are compared to MC prediction of Pythia MC09 and POWHEG. The Pythia MC09 results are scaled by a factor of 0.52 .

The measurement of the $b \bar{b}$ dijet cross section is also performed by estimating the number of events using template fits to the dijet invariant mass. Systematic uncertainties stemming from the used MC based templates have been taken into account in both measurements, but are negligible compared to the dominating uncertainties from $b$-tagging and the jet energy scale. The $b \bar{b}$ dijet 
cross section is shown in Fig. 3 as a function of the dijet mass $M$ compared to NLO predictions from POWHEG and to Pythia MC09. The Pythia MC09 results, again scaled by 0.52 , follow the shape of the measured cross section quite well, while POWHEG predicts a somewhat higher cross section at lower dijet masses.

\section{Conclusions}

The differential inclusive and dijet cross section of $b$-jets has been measured at ATLAS using the SV0 $b$-taging algorithm and compared to predictions of Pythia and with the NLO QCD calculations performed within a framework of the POWHEG generator. The measurement agrees with NLO prediction within systematic uncertainties that are dominated by uncertainties related to the the $b$-tagging efficiency and jet energy scale.

\section{References}

[1] The ATLAS Collaboration, JINST 3 (2008) S08003.

[2] T. Sjostrand, S. Mrenna and P. Skands, JHEP 05 (2006) 026.

[3] The ATLAS Collaboration, ATL-PHYS-PUB-2010-002.

[4] S. Alioli, K. Hamilton, P. Nason, C.Oleari and E. Re, arXiv:1012.3380v1.

[5] The ATLAS Collaboration, hep-ex/1109.6833v1 (2011).

[6] The ATLAS Collaboration, ATLAS-CONF-2011-011.

[7] The ATLAS Collaboration, ATLAS-CONF-2011-032.

[8] The ATLAS Collaboration, ATLAS-CONF-2010-99.

[9] M. Cacciari, G. P. Salam and G. Soyez, JHEP 04 (2008) 063.

[10] The ATLAS Collaboration, Eur. Phys. J. C71 (2011) 1512-1571. 